\title{
X-RAY LASERS, OPERATION AND APPLICATIONS
}

\author{
H. FIEDOROWICZ \\ Institute of Optoelectronics, Military University of Technology \\ Kaliskiego 2, 01-489 Warsaw, Poland
}

\begin{abstract}
$\mathrm{X}$-ray lasers are currently being studied in many laboratories around the world. This paper gives a short description of X-ray laser physics, presents the parameters of existing X-ray lasers and their applications. Research aimed at developing an efficient X-ray laser acceptable to potential users is presented in the final part of the paper.
\end{abstract}

PACS numbers: $42.60 . \mathrm{By}, 32.30 . \mathrm{Rj}$

\section{Introduction}

Since the first demonstration of soft X-ray lasing in 1985 [1, 2], significant progress has been made in X-ray lasers. Laser action has been observed at wavelengths as short as $3.5 \mathrm{~nm}$. The most successful $\mathrm{X}$-ray laser to date has been obtained using a collisional excitation of an active medium and a high power laser operated in the visible range as a pump source. Current research is focused on several fronts, however, the most important problem are improvement in gain efficiency, and the enhancement of brightness and coherence of X-ray laser beams. Many applications of X-ray lasers have been realized and expansion in this field can be anticipated. One may say that now we are transitioning from the discovery phase into the era of optimization and applications of X-ray lasers. Comprehensive information about achievements in this field during past few years can be found in the proceedings of a number of conferences on X-ray lasers [3-5] and in the technical papers referred therein.

\section{Physical background}

$\mathrm{X}$-ray lasers have been operated so far as simple amplifiers of spontaneous emission in a hot plasma column a few $\mathrm{cm}$ long and about $100 \mu \mathrm{m}$ in diameter. The plasma column is usually produced by irradiation of a target made from a thin foil, fiber or solid material with a high-power laser beam focused to a line. The appropriate X-ray laser physics is discussed in detail in a book by Elton [6]. Two principal schemes of excitation of an X-ray laser active medium are used. In the collision excitation scheme either neon-like or nickel-like ions are excited from the ground state ( $2 p$ for neon-like and $3 d$ for nickel-like ions) to the laser upper levels ( $3 p$ or $4 d$, respectively). As the laser lower levels ( $3 s$ or $4 p$ ) are radiatively 
coupled to the ground state, they are depopulated by resonance emission and population inversion between $3 p-3 s$ or $4 d-4 p$ is created. X-ray laser action in the recombination scheme requires a hot plasma, that contains highly ionized bare nuclei or helium-like ions. Rapid cooling during the hydrodynamic expansion of the plasma causes fast recombination, that leads to the population of excited states of hydrogen-like or lithium-like ions. The first excited state is depopulated by resonance emission given population inversion between the first and higher excited states. Typical temperatures of plasmas to obtain population inversion of highly charged ions are several hundred $\mathrm{eV}$ for the collision excitation scheme and several tens of $\mathrm{eV}$ for the recombination scheme. To heat plasmas to such temperatures power density of laser radiation producing the plasmas should exceed $10^{13} \mathrm{~W} \mathrm{~cm}^{-2}$ in the case of the collision scheme and $10^{12} \mathrm{~W} \mathrm{~cm}^{-2}$ in the case of the recombination scheme. It follows that the generation of an $\mathrm{X}$-ray laser requires a large laser facility to be able to produce subnanosecond laser pulses of energy in the range of several hundred joules up to several kilojoules.

\section{X-ray laser parameters}

Intensive research at many laboratories have worldwide demonstrated X-ray lasing at wavelengths ranging from 3.5 to $60 \mathrm{~nm}$ for both pumping schemes. X-ray laser beams of $\mathrm{MW}$ power and $\mathrm{mJ}$ energy in subnanosecond pulse have already been obtained. Thus far, the brightest X-ray laser in neon-like yttrium ions at $15.5 \mathrm{~nm}$ was obtained at the Lawrence Livermore National Laboratory, Livermore (LLNL) using the Nova laser. It emits radiation with a power of $32 \mathrm{MW}$ with an output pulse width of $200 \mathrm{ps}$ and an integrated energy of $7 \mathrm{~mJ}$ [7]. The yttrium $\mathrm{X}$-ray laser beam originates from an approximately $120 \mu \mathrm{m}$ diameter gain region and has a divergence $10 \mathrm{mrad}$. The line width of X-ray lasers narrows during amplification, because the gain is stronger at line center. For the neon-like selenium laser at $20.6 \mathrm{~nm}$ the line width is $1.0-1.2 \times 10^{-3} \mathrm{~nm}$ [8]. The longitudinal coherence length for the selenium laser estimated from the line width is about $300 \mu \mathrm{m}$. The spatial coherence of X-ray lasers measured with an uniformly redundant array of slits is approximately equal to the width of the line focus of the laser beam pumping the X-ray laser. A characteristic of X-ray lasers is the extraordinary brightness of the line emission. For the yttrium laser the estimated brightness was $4.6 \times 10^{17} \mathrm{~W} /\left(\mathrm{cm}^{2} \mathrm{sr} \mathrm{nm}\right)$, equivalent to the brightness of a $3-\mathrm{GeV}$ black body at this wavelength [7]. The output intensity was calculated to be $4.0 \times$ $10^{11} \mathrm{~W} / \mathrm{cm}^{2}$ at the peak of the pulse. Polarization of the X-ray laser beam in a neon-like germanium plasma has been observed [9]. In summary, typical X-ray laser characteristics available today are listed below:

$\begin{array}{ll}\text { laser wavelengths } & 3.5-60 \mathrm{~nm} \\ \text { output power } & 0.1-10 \mathrm{MW} \\ \text { output energy } & \mu \mathrm{J}-\mathrm{mJ} \\ \text { divergence } & 1-10 \mathrm{mrad} \\ \text { longitudinal coherence } & \approx 100 \mu \mathrm{m} \\ \text { spatial coherence } & \approx 100 \mu \mathrm{m} .\end{array}$




\section{X-ray laser applications}

Current applications of X-ray lasers exploit their extremely high brightness. These applications include imaging of biological objects and imaging of rapid processes in laser fusion plasmas. X-ray imaging of biological microstructures using $\mathrm{X}$-ray lasers is a natural extension of a technique developed for synchrotron sources. Microscopy with short pulse ( $<1 \mathrm{~ns})$ X-ray lasers offers the opportunity for high resolution imaging of biological specimens in their natural environment without the blurring effects associated with natural motion and radiation induced damage. Additionally, the narrow spectral width of the X-ray laser is well matched to zone plates and multilayer mirrors used in X-ray imaging microscopy. The first image of a biological cell, obtained with an X-ray laser with submicron $(\approx 0.1 \mu \mathrm{m})$ resolution, was made using a contact microscopy technique and the hydrogen-like carbon recombination laser at $18.2 \mathrm{~nm}$ [10]. Direct X-ray images of biological cells have been made at the LLNL using a nickel-like tantalum X-ray laser at $4.48 \mathrm{~nm}$, generated with the Nova laser [11]. In this first X-ray laser microscope operating within the "water window" between the $K$-absorption edges of carbon and oxygen [12] the X-ray laser beam was collected and focused onto a specimen with a spherical tungsten-carbide/carbon multilayer coated X-ray mirror. The narrow band pass of the mirror serves to eliminate the background X-ray continuum. The specimen to be imaged is deposited onto a silicon nitride window (100 nm thick) and placed at the focus of the spherical mirror. The illuminated specimen is imaged onto a microchannel plate detector by using an X-ray zone plate lens. An estimated resolution of the microscope was $\approx 55 \mathrm{~nm}$. Since most biological microstructures of interest are three-dimensional in nature, it is evident that in order to increase the ability of X-ray microscopy three-dimensional imaging has to be developed. A variety of techniques to achieve high-resolution three-dimensional imaging with the use of X-ray lasers have been discussed. They included X-ray holography [13] and X-ray tomography [14]. X-ray laser beams are now being used as density diagnostics for laser-produced plasma experiments. The techniques used in these studies involve X-ray interferometry [15] and X-ray radiography [16]. The first X-ray interferometer developed at the LLNL uses a neon-like yttrium X-ray laser at $15.5 \mathrm{~nm}$. The system consists of a Mach-Zehnder interferometer with two flat multilayer mirrors, two multilayer beam splitters, and an imaging multilayer mirror. The multilayer optics components were designed and fabricated to reflect $15.5 \mathrm{~nm}$ laser wavelength at a near normal incidence with reflectivity about $60 \%$. The X-ray image was registered with a back illuminated charge coupled device (CCD) detector. The X-ray interferometer was applied to study high-density laser-produced plasmas [17].

In addition to these two application techniques there are numerous possible applications of X-ray lasers in atomic and molecular physics, surface physics and chemistry, material science and current technology, that can be expected to complement the research potential of synchrotron radiation. A comprehensive discussion of this subject can be found in the proceedings of the $1992 \mathrm{X}$-ray lasers application workshop in San Francisco [18] and in numerous review papers [19, 20]. 


\section{New trends in X-ray laser research}

Existing X-ray lasers have been generated in conjunction with large laser facilities, which are built mainly for laser fusion studies. Future applications of X-ray lasers by a larger community of scientists depend on the possibility of developing a short wavelength, highly efficient, low cost, and small size X-ray laser. Efforts to obtain such laser are underway. Current research is proceeding on two fronts. One major effort puts emphasis on the improvement of the efficiency and brightness of the existing collisional excitation lasers by using pulse-shaping [21], pre-pulse [22], or multi-pulse [23] pumping of the gain medium, and by using a curved slab target [24]. The multi-pulse pumping facilitates the production of suitable density gradients of the plasma in a gain medium, the curved target compensates for the refraction of the X-ray laser beam on the strong density gradients. These techniques significantly improve the characteristics of the existing X-ray lasers [25-27]. It is expected that these methods will allow the development of a small-scale collisionally excited X-ray laser [28]. Enhancement of brightness and coherence was also obtained by using an injector/amplifier double target configuration [29], and traveling wave excitation [30]. Other approaches are based on improving the $\mathrm{X}$-ray recombination laser by using a specially designed target surrounded with metal blades to increase radiation cooling [31] or a capillary target to confine the plasma column [32], or by using high-intensity short-pulse driving lasers to excite the gain medium [33, 34]. Advances in subpicosecond laser technology in the last few years have led to the availability of small-scale laser with focused power densities exceeding $10^{18}-10^{20} \mathrm{~W} \mathrm{~cm}^{-2}$. With such lasers two proposed $\mathrm{X}$-ray laser schemes, based on direct optical-field-ionization (OFI) by laser radiation [35], or based on inner-shell-photoionization (ISPI) by laser-produced X-rays [36], seems to be possible. Soft X-ray amplification by OFI has been demonstrated [37], however, the ionization-induced refraction of the X-ray laser beam associated with the confocal geometry limits the gain [38]. It is likely that this problem will be solved with the plasma channel approach [39] to channel an X-ray laser beam with a wave guide during amplification. The ISPI scheme, which requires a higher driving laser energy, gives the possibility of $\mathrm{X}$-ray lasing in a very short-wavelength range $(\lambda \leq 1.5 \mathrm{~nm})$. A femtosecond high-power laser has been also applied to obtain a collisional excitation X-ray laser at $41.8 \mathrm{~nm}$ with palladium-like xenon ions [40].

In addition to these efforts, we propose to use a gas puff target instead of a solid target to create an X-ray laser active medium [41]. The gas puff target obtained by pulsed injection of gas into the laser focus offers the possibility of forming a gain medium with a flat density profile of appropriate maximum density and should improve the characteristics of X-ray lasers. Other advantages of the gas puff target are the possibility of working at a high repetition rate and the absence of debris emitted from the laser-irradiated target. Using the gas puff target, X-ray lasing with neon-like argon at $46.9 \mathrm{~nm}$ and nickel-like xenon ions at $10.0 \mathrm{~nm}$ were observed for the first time [42]. A new X-ray laser excitation scheme with the resonant self-photopumping of neon-like ions also has been demonstrated [43]. 
Alternatively investigated to laser-driven $\mathrm{X}$-ray lasers, discharge-pumped $\mathrm{X}$-ray lasers may, in the future, significantly increase efficiency and hence reduce the costs of these devices. However, up to now, only lasing at $46.9 \mathrm{~nm}$ in neon-like argon ions for collisional excitation X-ray laser [44] has been demonstrated.

\section{Conclusions}

At present, the X-ray laser is the brightest laboratory source of soft X-rays, it offers unique possibilities for applications in a variety of scientific and technical areas, and it is complementary to synchrotrons. However, many problems must be solved before X-ray lasers become popular. First of all, the efficiency of X-ray generation should be considerably increased. Strong efforts are being expanded in this direction and the development of low cost tabletop size X-ray lasers seems to be possible in near future. In spite of the fact that X-ray laser technology is relatively new, it is already being applied, most notably to X-ray microscopy of biological microstructures and X-ray interferometry of laser plasmas. Other applications of $\mathrm{X}$-ray lasers to science and technology have been discussed and proposed.

\section{Acknowledgments}

The author wishes to thank E. Fill, G. Jamelot, D. Matthews, J. Nilsen, B. Rus, L.B. DaSilva, and S. Suckewer for valuable discussions and A. Bartnik, P. Celliers, V.M. Dyakin, A.Ya. Faenov, J. Kostecki, Y. Li, P. Lu, A. Morozov, P. Parys, G. Pretzler, S. Sebban, I.Yu. Skobelev, and M. Szczurek for the co-operation in performing $\mathrm{X}$-ray laser experiments. This work was supported, in part, by the NATO Collaborance Research grant HTECH CRG 950396.

\section{References}

[1] D.L. Matthews, P.L. Hagelstein, M.D. Rosen, M.J. Eckart, N.M. Ceglio, A.U. Hazi, H. Medecki, B.J. MacGovan, J.E. Trebes, B.L. Whitten, E.M. Campbell, C.W. Hatcher, A.M. Hawryluk, R.L. Kauftman, L.D. Pleasance, G. Rambach, J.H. Scofield, G. Stone, T.A. Weaver, Phys. Rev. Lett. 54, 110 (1985).

[2] S. Suckewer, C.H. Skinner, H. Milchberg, C. Keane, D. Vorhees, Phys. Rev. Lett. 54, 106 (1985).

[3] X-ray Lasers 1990, Ed. G.J. Tallents, IOP Publishing, Bristol 1990.

[4] $X$-ray Lasers 1992, Ed. E.E. Fill, IOP Publishing, Bristol 1992.

[5] X-ray Lasers 1994, Eds. D.C. Eder, D.L. Matthews, AIP Press, New York 1994.

[6] R.C. Elton, X-ray Lasers, Academic Press, New York 1990.

[7] L.B. DaSilva, B.J. MacGovan, S. Mrowka, J.A. Koch, R.A. London, D.L. Matthews, J.H. Underwood, Opt. Lett. 18, 1174 (1993).

[8] B.J. MacGovan, L.B. DaSilva, D.J. Fields, C.J. Keane, J.A. Koch, R.A. London, D.L. Matthews, S. Maxon, S. Mrowka, A.L. Osterheld, J.H. Scofield, G. Shimka veg, J.E. Trebes, R.S. Walling, Phys. Fluids B 4, 2326 (1992).

[9] B. Rus, C.L.S. Lewis, G.F. Cairns, P. Dhez, P. Jaeglé, M.H. Key, D. Neely, A.G. MacPhee, S.A. Ramsden, C.G. Smith, A. Sureau, Phys. Rev. A 51, 2316 (1995).

[10] C.H. Skinner, D.S. DiCicco, D. Kim, R.J. Rosser, S. Suckewer, A.P. Gupta, J.G. Hirschberg, J. Microsc. 159, 51 (1990). 
[11] L.B. DaSilva, J.E. Trebes, R. Balhorn, S. Mrowka, E. Anderson, D.T. Attwood, T.W. Barbee, Jr., J. Brase, M. Corzett, J. Gray, J.A. Koch, C. Lee, D. Kern, R.A. London, B.J. MacGovan, D.L. Matthews, G. Stone, Science 258, 269 (1992).

[12] L.B. DaSilva, J.E. Trebes, S. Mrowka, T.W. Barbee, Jr., J. Brase, J.A. Koch, R.A. London, B.J. MacGovan, D.L. Matthews, G. Stone, T. Yorkey, E. Anderson, D.T. Attwood, D. Kern, Opt. Lett. 17, 754 (1992).

[13] H. Daido, M.S. Schulz, K. Murai, R. Kodama, G. Yuan, J. Goto, K.A. Tanaka, Y. Kato, S. Nakai, K. Shinohara, T. Honda, I. Kodama, H. Iwasaki, T. Yoshinobu, M. Tsukamoto, M. Niibe, Y. Fukuda, D. Neely, A. MacPhee, G. Slark, J. X-ray Sci. Technol. 5, 105 (1995).

[14] W.S. Haddad, I. McNulty, J.E. Trebes, E.H. Anderson, L. Yang, J.M. Brase, in: $X$-ray Lasers 1994, Eds. D.C. Eder, D.L. Matthews, AIP Press, New York 1994, p. 559.

[15] L.B. DaSilva, T.W. Barbee, Jr., R. Cauble, P. Celliers, D. Ciarlo, S. Libby, R.A. London, D. Matthews, S. Mrowka, J.C. Moreno, D. Ress, J.E. Trebes, A.S. Wan, F. Weber, Phys. Rev. Lett. 74, 3991 (1995).

[16] R. Cauble, L.B. DaSilva, T.W. Barbee, Jr., P. Celliers, J.C. Moreno, A.S. Wan, Phys. Rev. Lett. 74, 3816 (1995).

[17] A.S. Wan, L.B. DaSilva, T.W. Barbee, Jr., R. Cauble, P. Celliers, S.B. Libby, R.A. London, J.C. Moreno, J.E. Trebes, F. Weber, J. Opt. Soc. Am. B 13, 447 (1996).

[18] Applications of $X$-ray Lasers, Eds. R. London, D.L. Matthews, S. Suckewer, National Technical Information Service, Springfield (VA) 1992.

[19] B. Crasemann, in: X-ray Lasers 1994, Eds. D.C. Eder, D.L. Matthews, AIP Press, New York 1994, p. 566.

[20] C.D. Caldwell, in: X-ray Lasers 199/, Eds. D.C. Eder, D. Matthews, AIP Press, New York 1994, p. 579.

[21] S. Maxon, K.G. Estabrook, M.K. Prasad, A.L. Osterheld, R.A. London, D.C. Eder, Phys. Rev. Lett. 70, 2285 (1993).

[22] J. Nilsen, J.C. Moreno, B.J. MacGovan, J.A. Koch, Appl. Phys. B 57, 309 (1993).

[23] H. Daido, R. Kodama, K. Murai, G. Yuan, M. Takagi, Y. Kato, I.W. Choi, C.H. Nam, Opt. Lett. 29, 61 (1995).

[24] R. Kodama, D. Neely, Y. Kato, H. Daido, K. Murai, G. Yuan, A. MacPhee, C.L.S. Lewis, Phys. Rev. Lett. 73, 3215 (1994).

[25] J. Nilsen, J.C. Moreno, Phys. Rev. Lett. 74, 3376 (1995).

[26] E.E. Fill, Y. Li, D. Schlögl, J. Steingruber, Opt. Lett. 20, 374 (1995).

[27] H. Daido, Y. Kato, K. Murai, S. Ninomiya, R. Kodama, G. Yuan, Y. Oshikane, M. Takagi, H. Takabe, Phys. Rev. Lett. 75, 1074 (1995).

[28] S. Basu, P.L. Hagelstein, J.G. Goodberlet, M.H. Muendel, S. Kaushik, Appl. Phys. $B$ 57, 303 (1993).

[29] C.L.S. Lewis, D. Neely, D.M. O'Neil, J.O. Uhomoibhi, M.H. Key, Y. Al Hadithi, G.J. Tallents, S.A. Ramsden, Opt. Commun. 91, 71 (1992).

[30] J.C. Moreno, J. Nilsen, L.B. DaSilva, Opt. Commun. 110, 585 (1994).

[31] C.O. Park, L. Polonsky, S. Suckewer, Appl. Phys. B 58, 19 (1994).

[32] S. Suckewer, to be published. 
[33] J.C. Solem, T.S. Luk, K. Boyer, C.K. Rhodes, IEEE J. Quantum Electron. 25, 2423 (1989).

[34] J. Zhang, M.H. Key, Appl. Phys. B 58, 13 (1994).

[35] N.H. Burnett, G.D. Enright, IEEE J. Quantum Electron. 26, 1797 (1990).

[36] H. Kapteyn, Appl. Opt. 31, 4931 (1992).

[37] Y. Nagata, K. Midorikawa, S. Kubodera, M. Obara, H. Tashiro, K. Toyoda, Phys. Rev. Lett. 71, 3774 (1993).

[38] D.C. Eder, P. Amendt, L.B. DaSilva, R.A. London, B.J. MacGovan, D.L. Matthews, B.M. Penetrante, M.B. Rosen, S.C. Wilks, T.D. Donnelly, R.W. Falcone, G.L. Strobel, Phys. Plasmas 1, 1744 (1994).

[39] H.M. Milchberg, C.G. Durfee III, J. Lynch, J. Opt. Soc. Am. B 12, 731 (1995).

[40] B.E. Lemoff, G.Y. Yin, C.L. Gordon III, C.P.J. Barty, S.E. Harris, Phys. Rev. Lett. 74, 1574 (1995).

[41] H. Fiedorowicz, A. Bartnik, K. Gac, P. Parys, M. Szczurek, J. Tyl, in: X-ray Lasers 1994, Eds. D.C. Eder, D.L. Matthews, AIP Press, New York 1994, p. 538.

[42] H. Fiedorowicz, A. Bartnik, Y. Li, P. Lu, E. Fill, Phys. Rev. Lett. 76, 415 (1996).

[43] J. Nilsen, H. Fiedorowicz, A. Bartnik, Y. Li, P. Lu, E.E. Fill, Opt. Lett. 21, 410 (1996).

[44] J.J. Rocca, V. Shlyaptsev, F.G. Tomasel, O.D. Cortazar, D. Hartshorn, J.L. Chilla, Phys. Rev. Lett. 73, 2192 (1994). 